\title{
Comparison of the Effects of Individual and Group Horticulture Interventions
}

\section{Yuka Kotozaki*}

Smart Ageing International Research Center, Institute of Development, Aging, and Cancer, Tohoku University, Sendai, Japan

\begin{abstract}
Chronic stress adversely affects the body, and stress and negative emotions affect the development and progression of diseases. This study focuses on horticultural therapy (HT) as a method of stress reduction. Although previous studies have reported that HT has many benefits, the effects of HT in relation to differences in the intervention style have not been investigated. The purpose of this study was to clarify whether there is a difference in the effect due to the difference in intervention style in HT. The participants were divided into three groups, a group intervention (GI group; $n=15)$, an individual intervention (II group; $n=15$ ), and a control group (C group; $n=15$ ). The GI and II groups underwent four weeks of a horticultural intervention, whereas the $C$ group was provided with a gardening kit by an experimenter. The individuals in the $\mathrm{C}$ group cared for the plants by themselves for $15 \mathrm{~min}$ per day for one month. The Gl group showed significant improvement in the WHO Quality of Life 26 (WHO-QOL26) subscore, the Emotional Intelligence Scale (EQS) subscore, the General Health Questionnaire (GHQ) score, and salivary cortisol level, as compared with the II group. These findings suggest that a group HT intervention might be more effective than an individual intervention.
\end{abstract}

Keywords: Horticultural therapy; Group intervention; Individual intervention

\section{Introduction}

Many Japanese people lead stressful lives, as do many people in other parts of the world. Previous studies suggest that the chronic stress adversely affects the body [1] and that stress and negative emotions can affect the development and progression of diseases [2]. The reduction of stress is important for maintaining and enhancing health. In recent years, methods of stress relief have been sought by many researchers. This study focuses on horticultural therapy (HT).

HT is a method of psychological care for treating post-traumatic stress disorder (PTSD) that was developed in the United States after World War II for the psychological care and social rehabilitation of disabled soldiers and war veterans showing PTSD symptoms [3]. HT interventions are led by professionals trained to incorporate the use of plants and horticultural education into rehabilitation therapies [3]. It has been reported that participants begin to identify with plant growth, and regain health and motivation. Through such experiences and their association with nature, participants are thought to experience improvement [4]. HT has mainly been developed for elderly adults and people with disabilities [5,6]. Previous studies have suggested that HT and exposure to nature can have cognitive [7,8], psychological [3,912], social [13,14], and physical benefits [10]. It has also been suggested that HT has a positive effect on physiological factors, such as heart rate and salivary cortisol levels [15]. Previous studies have reported many therapeutic effects of HT in care and education programs for disabled patients and the elderly $[12,14,16-19]$. However, the effects of HT in relation to difference in the intervention style, such as group versus individual interventions, have not previously been investigated.

The purpose of this study was to clarify whether there is a difference in the effect of HT in relation to a difference in the style of the HT intervention, using psychological measures and salivary cortisol level. We hypothesized that a group HT intervention may produce better psychological effects than an individual intervention. Although the content of the intervention was different from that used in previous studies, it has been reported that group interventions may produce greater improvement than individual interventions [20].

\section{Materials and Methods}

\section{Participants}

Forty-five healthy, right-handed university students or postgraduates ( 22 men and 23 women; age, $21.22 \pm 2.42$ years) participated in this study. They had normal vision and none had a history of neurological or psychiatric illness. Written informed consent was obtained from each participant in accordance with the Declaration of Helsinki (1991). Then, they were randomly allocated into group intervention (GI), individual intervention (II), and control (C) groups. The study was approved by the Ethics Committee of Tohoku University School of Medicine.

\section{Procedure}

Participants who were assigned to the GI and II groups participated in a horticultural intervention in the laboratory at a specified date and time. Participants in the GI group took the horticultural intervention in groups of five (total 3 groups). Before the start of the intervention, all participants were assessed on the basis of some psychological measures. The horticultural intervention was designed in collaboration with a horticultural therapist and clinical psychologists. This intervention comprised a total of four weekly sessions (60 min each) at a university lab and 15 min per day at participants' homes. The sessions at the university lab comprised interactive lectures and practical horticultural training. Participants attended four horticultural lessons, including topics such as designing a garden planter, seeding, watering, weeding, and picking flowers. They filled out a horticultural intervention session

${ }^{*}$ Corresponding author: Yuka Kotozaki, Smart Ageing International Research Center Institute of Development, Aging and Cancer, Tohoku University, 4-1 Seiryo-machi, Aobaku, Sendai 980-8575, Japan, Tel: 81 (0) 22717 7988; Fax: 81 (0) 22717 7988; E-mail: kotoyuka@idac.tohoku.ac.jp

Received December 23, 2013; Accepted February 27, 2014; Published March 1, 2014

Citation: Kotozaki Y (2014) Comparison of the Effects of Individual and Group Horticulture Interventions. Health Care Current Reviews 2: 120. doi: 10.4172/ hccr. 1000120

Copyright: (c) 2014 Kotozaki Y. This is an open-access article distributed under the terms of the Creative Commons Attribution License, which permits unrestricted use, distribution, and reproduction in any medium, provided the original author and source are credited. 
checklist after each session as self-assessment. Participants took care of plants for 15 min per day at their convenience, using horticulture kits provided by the experimenters, and recorded the completion of this task daily on forms provided by the experimenters at the intervention sessions. The participants submitted these forms to the experimenters at the weekly horticultural intervention sessions. Participants who were assigned to the $\mathrm{C}$ group were provided with a gardening kit by an experimenter; they cared for the plants by themselves for $15 \mathrm{~min}$ per day for one month.

\section{Psychological measures}

We used Japanese versions of the following psychological measures.

Assessment of quality of life: The World Health Organization Quality of Life 26 (WHO-QOL26) is a 26-item, self-report measure designed to assess quality of life [21]. Twenty-four items measure the four domains of QOL: physical, psychological, social, and environmental, and the other two items measure overall QOL and general health. The score for each question ranges from 1 to 5 , with higher scores reflecting higher QOL. The present study used the Japanese version of the WHO-QOL26 [21].

Assessment of depressive symptoms: The Center for Epidemiologic Studies Depressive Symptoms Scale (CES-D) is a 20item, self-report measure designed to assess depressive symptoms $[22,23]$. Scores for each item are summed to give a range of total scores from 0 to 60 . A higher score indicates a greater tendency toward depressive symptoms. A score of 16 points or higher suggests the presence of clinical depressive symptoms. The reliability and validity of the Japanese version of the CES-D have been confirmed [23]. In the Japanese version, the cutoff value of 16 was also optimal, as assessed by comparing the proportion of patients with CES-D scores of 16 points or higher in a normal control group with that in a group of patients with mood disorders [23].

Assessment of emotional intelligence: The Japanese version of the Emotional Intelligence Scale (EQS) is a 65-item, self-report measure designed to assess emotionally intelligent behavior, which provides an estimate of one's underlying emotional and social intelligence [2426]. The scale was developed and standardized for use with Japanese subjects. A more detailed discussion of the psychometric properties of this instrument and how it was developed is found in the Emotional Intelligence Scale technical manual [26]. The participant's responses render the following three composite scale scores (factors): (a) Intrapersonal factor (comprising self-insight, self-motivation, and self-control), (b) Interpersonal factor (comprising empathy, altruism, and interpersonal control), and (c) Situation Management factor (comprising insight into and control of a situation). Each composite scale score comprises three subscale scores. All three factors of the EQS have been shown to be associated with better mental health, as measured by the General Health Questionnaire (GHQ). The Situation Management factor has been shown to be strongly associated with better mental health [24]. This result suggests that higher emotional intelligence leads to better mental health [27].

Assessment of mental health: The GHQ is a 30 -item selfreport measure designed to assess mental health [28,29]. This scale includes six subscales: "general illness," "somatic symptoms," "sleep disturbance," "social dysfunction," "anxiety and dysthymia," and "suicidal depression." The questionnaire uses a four-point Likert scoring method. The total score for the GHQ-30 is six or lower in $85 \%$ of healthy adults; in this study, we used only the total score.
Assessment of mood state: The Profile of Mood States (POMS) is a 65 -item self-report measure designed to assess mood states [30,31]. It consists of the following six mood state scales: tension-anxiety $(\mathrm{T}-\mathrm{A})$, depression-dejection $(\mathrm{D})$, anger-hostility $(\mathrm{A}-\mathrm{H})$, fatigue $(\mathrm{F})$, confusion $(\mathrm{C})$, and vigor $(\mathrm{V})$. The reliability and validity of the POMS have been examined in the Japanese population [31].

\section{Saliva sampling}

We collected saliva samples from participants to measure their salivary cortisol levels. Distressing psychological stimuli are associated with an increased cortisol level [32,33]. Considering the participants' circadian cortisol rhythms, we collected all saliva samples at 4:00 pm on weekdays, before and after the intervention. We selected 4:00 pm because humans are less affected by circadian cortisol rhythms at this time of day [34]. Participants were asked to refrain from drinking, eating [35], and exercising [36] for two hours before saliva sampling. This method was same as that in our previous studies [32,37].

\section{Measurement of salivary cortisol}

To assess physiological stress, we employed the same technique to measure salivary cortisol as described in a previous study $[32,37]$. Saliva samples were collected using the Salivette apparatus (Sarstedt, Nümbrecht, Germany). Cortisol was measured in the supernatant solutions, which were stored in airtight containers at $-80^{\circ} \mathrm{C}$. We measured salivary cortisol with a semi-microcolumn high-performance liquid chromatography (HPLC) system (Shiseido, Tokyo).

\section{Analytical methods}

The psychological and salivary data were analyzed using the PASW statistical software package (ver. 18 for Windows; SPSS Inc., Chicago, IL, USA). To examine the psychological effects, a mixed design was used to compare the difference between the three groups pre- and postintervention. Additionally, as our primary endpoint of interest was the beneficial effect of intervention training, test-retest changes were compared between the intervention and control groups using onetailed tests $(\mathrm{p}<0.05)$, in the same manner as in previous studies [32,37].

\section{Results}

\section{Differences between three groups}

The participants' demographic data are shown in Table 1; the ages of the three groups did not differ significantly. Comparisons of the psychological changes pre- and post-intervention are shown in Table 2. The GI group showed significant improvement, relative to the $\mathrm{C}$ group, in the WHO-QOL26 Psychological score $[\mathrm{F}(2,42)=4.37, p<0.01]$, the WHO-QOL26 Social score $[\mathrm{F}(2,42)=4.76, \mathrm{p}<0.01]$, the EQS Interpersonal score $[\mathrm{F}(2,42)=2.80, p<0.05]$, the EQS Empathy score $[\mathrm{F}(2,42)=4.38, p<0.01]$, and the EQS Altruism score $[\mathrm{F}(2,42)=3.24$, $\mathrm{p}<0.05]$. Furthermore, the GI group showed a significant decrease, relative to the $\mathrm{C}$ group, in the GHQ score $[\mathrm{F}(2,42)=2.66, p<0.05]$ and POMS vigor score $[\mathrm{F}(2,42)=2.45, p<0.05]$. Additionally, the GI group showed a significant decrease in salivary cortisol level compared with the $\mathrm{C}$ group $[\mathrm{F}(2,42)=5.03, p<0.01]$. The II group did not differ significantly from the $\mathrm{C}$ group.

\section{Comparison of pre- and post-intervention scores in the GI and II groups}

Comparisons of the psychological changes pre- and postintervention between the GI and II groups are shown in Table 2. Relative to the II group, the GI group showed a significantly higher 
Citation: Kotozaki Y (2014) Comparison of the Effects of Individual and Group Horticulture Interventions. Health Care Current Reviews 2: 120. doi: $10.4172 / 2375-4273.1000120$

Page 3 of 5

\begin{tabular}{|l|l|l|l|l|l|l|l|}
\hline & Gl group $(\mathrm{N}=15)$ & II group $(\mathrm{N}=15)$ & C group $(\mathrm{N}=15)$ & & & \\
\hline Factor & Mean & SD & Mean & SD & Mean & SD \\
\hline Age & 20.53 & 2.45 & 21.60 & 1.54 & 21.53 & 3.00 \\
\hline
\end{tabular}

aOne-way analysis of variance.

Gl, group intervention; II, individual intervention; C, control; SD, standard deviation

Table 1. Demographic data of the participants

\begin{tabular}{|c|c|c|c|c|c|c|c|c|c|c|c|c|}
\hline & GI group & II group & & & C group & & & & & & & \\
\hline & Pre & Post & Pre & & Post & & Pre & Post & & & & \\
\hline Measures & Mean & SD & Mean & SD & Mean & SD & Mean & SD & Mean & SD & Mean & SD \\
\hline WHO-QOL26 & 3.23 & 0.53 & 2.93 & 0.58 & 3.23 & 0.4 & 3.01 & 0.69 & 3.07 & 0.57 & 3.18 & 0.55 \\
\hline \multicolumn{13}{|l|}{ Physical QOL score } \\
\hline Psychological score & 2.92 & 0.53 & 3.38 & 0.4 & 3.03 & 0.56 & 2.92 & 0.68 & 2.82 & 0.45 & 2.82 & 0.41 \\
\hline Social score & 3.18 & 0.89 & 3.75 & 0.8 & 3.53 & 0.75 & 3.58 & 0.65 & 3.4 & 0.62 & 3.27 & 0.61 \\
\hline Environmental score & 3.39 & 0.46 & 3.22 & 0.47 & 3.31 & 0.47 & 3.27 & 0.32 & 3.02 & 0.37 & 3.07 & 0.37 \\
\hline Global score & 3.24 & 0.56 & 3.35 & 0.45 & 3.32 & 0.49 & 3.3 & 0.42 & 3.17 & 0.42 & 3.19 & 0.43 \\
\hline CES-D & 13.53 & 10.32 & 7.67 & 3.64 & 9.53 & 5.28 & 8.87 & 6.67 & 14 & 11.77 & 13.73 & 4.04 \\
\hline EQS & 46.6 & 11.87 & 49 & 13.52 & 54.67 & 11.88 & 56.93 & 12.5 & 42.4 & 7.43 & 46.4 & 9.3 \\
\hline \multicolumn{13}{|l|}{ Intrapersonal } \\
\hline Self-awareness & 12.07 & 3.65 & 12.73 & 4.06 & 15.47 & 4.93 & 16.33 & 4.55 & 11.33 & 3.7 & 12.33 & 3.92 \\
\hline Self-motivation & 14.13 & 5.3 & 14.8 & 5.35 & 17.47 & 4.5 & 17.93 & 4.28 & 13.2 & 2.81 & 14.47 & 3.7 \\
\hline Self-control & 20.4 & 4.52 & 21.47 & 6.6 & 21.73 & 6.32 & 22.67 & 6.11 & 17.87 & 4.73 & 19.6 & 4.5 \\
\hline Interpersonal & 41.87 & 12.8 & 46.8 & 11.1 & 44.4 & 13.73 & 42.67 & 7.54 & 47.8 & 12.14 & 46.33 & 12.27 \\
\hline Empathy & 13.67 & 4.12 & 15.53 & 3.4 & 16.53 & 5.57 & 14.87 & 3.62 & 15.87 & 3.6 & 15.87 & 3.46 \\
\hline Altruism & 12.6 & 4.4 & 14.27 & 4.43 & 12.2 & 4.9 & 13.2 & 4.72 & 16.07 & 4.32 & 14.8 & 5.25 \\
\hline Interpersonal relationship & 15.6 & 6.7 & 17 & 5.77 & 15.67 & 6.95 & 14.6 & 3.52 & 15.87 & 6.44 & 15.67 & 5.89 \\
\hline Situational & 41.07 & 15.21 & 43.93 & 14.23 & 43.07 & 15.07 & 44 & 12.61 & 36.87 & 11.11 & 36.13 & 10.45 \\
\hline Situational awareness & 18.53 & 6.65 & 19.13 & 5.78 & 18.4 & 8.64 & 19 & 6.11 & 17.07 & 4.28 & 16.6 & 5.49 \\
\hline Leadership & 10.27 & 5.19 & 12.07 & 5.24 & 10.67 & 5.19 & 11.33 & 4.84 & 8.53 & 4.1 & 8.53 & 3.93 \\
\hline Flexibility & 12.27 & 4.1 & 12.73 & 4.33 & 14 & 4.12 & 13.67 & 3.39 & 11.27 & 3.79 & 11 & 3.93 \\
\hline GHQ score & 6 & 3.34 & 3.2 & 2.81 & 6.07 & 3.88 & 4.33 & 3.5 & 4.73 & 2.79 & 5.47 & 5.97 \\
\hline POMS Tension-Anxiety & 6.53 & 4.42 & 6.07 & 2.89 & 8.73 & 4.32 & 7.4 & 4.27 & 8.2 & 4.9 & 7.93 & 4.35 \\
\hline Depression & 5.87 & 5.58 & 2.73 & 2.55 & 6.4 & 4.03 & 4.87 & 3.85 & 5.67 & 6.5 & 5.6 & 5.96 \\
\hline Anger-Hostility & 4.07 & 3.35 & 4 & 3.89 & 4.93 & 4.11 & 5.07 & 4.13 & 5 & 4.84 & 3.87 & 3.16 \\
\hline Vigor & 8.87 & 3.52 & 10.47 & 3.72 & 8.73 & 4.42 & 9.93 & 3.9 & 9.73 & 4.04 & 9 & 4.02 \\
\hline Fatigue & 8.27 & 4.1 & 5.73 & 3.41 & 8.2 & 3.88 & 6.13 & 3.2 & 8.8 & 5 & 7.07 & 5.23 \\
\hline Confusion & 7.2 & 3.38 & 6.33 & 2.5 & 6.6 & 2.87 & 5.87 & 3.2 & 6.47 & 4.42 & 6.27 & 4.06 \\
\hline Total Mood Disturbance & 23.07 & 19 & 14.4 & 13.68 & 26.13 & 15.25 & 19.4 & 15.78 & 24.4 & 22.03 & 21.73 & 19.87 \\
\hline Salivary cortisol level & 3.96 & 0.96 & 2.15 & 0.79 & 4.54 & 3.21 & 3.97 & 1.74 & 3.97 & 1.41 & 4.21 & 0.94 \\
\hline
\end{tabular}

a One-way analyses of covariance with pre-post differences in psychological measures as dependent variables and pre-intervention scores as covariates (one-tailed). GI, group intervention; II, individual intervention; C, control; SD, standard deviation; WHO-QOL26, World Health Organization Quality of Life 26; CES-D, Center for epidemiologic studies depression scale; EQS, Emotional Intelligence Scale; GHQ, The General Health Questionnaire; POMS, Profile of Mood States.

Table 2. Psychological measures pre- and post-intervention

post-intervention WHO-QOL26 Psychological score $[\mathrm{F}(1,28)=5.92$, $p<0.05]$, WHO-QOL26 Social score $[\mathrm{F}(1,28)=3.97, p<0.05]$, EQS Interpersonal score $[\mathrm{F}(1,28)=4.15, p<0.05]$, and EQS Empathy score $[\mathrm{F}(1,28)=7.97, \mathrm{p}<0.005]$. The GI group also showed a significantly lower post-intervention GHQ score $[\mathrm{F}(1,28)=3.05, p<0.05]$ than the II group. Additionally, the GI group exhibited a significantly lower salivary cortisol level $[\mathrm{F}(1,28)=2.93, p<0.05]$ than the II group.

\section{Discussion}

The purpose of this study was to investigate whether there is a difference in the effects of an HT intervention due to the difference in intervention style, using psychological measures and salivary cortisol level. The study revealed that the GI group showed improved psychological measures (WHO-QOL26, EQS, GHQ, and POMS) and salivary cortisol levels post-intervention compared with the other two groups. Additionally, the GI group was also showed improved psychological measures (WHO-QOL26, EQS, and GHQ) and salivary cortisol levels post-intervention compared with the II group. These results are consistent with our hypothesis that HT may be more effective by group intervention compared with individual intervention.

The GI group showed improved WHO-QOL26 scores (psychological score and social score) than the II and C groups, indicating that the group HT intervention increased psychological and social QOL more than the individual intervention. Previous studies have reported that HT improved QOL $[11,37,38]$. The raising of plants in a group is thought to have brought new hope and stimulation to the participants, and this may have led to greater improvement of their QOL (in particular, psychological and social aspects), relative to an individual intervention, by synergy. The GI group showed improved EQS scores (interpersonal, empathy, and altruism scores) relative to the II and C groups, indicating that the group HT intervention increased interpersonal intelligence more than the individual intervention. Previous studies have suggested that HT improves emotional intelligence $[39,40]$. Conducting a multiple activity in a group is thought 
Citation: Kotozaki Y (2014) Comparison of the Effects of Individual and Group Horticulture Interventions. Health Care Current Reviews 2: 120. doi: $10.4172 / 2375-4273.1000120$

to have developed a sense of community, interpersonal relationship, empathy, altruism, and so on. These effects were reflected more in the EQS interpersonal factor score of participants in the GI group than the II group. The GI group showed improved GHQ scores in comparison with the other two groups, indicating that the group HT intervention improved mental health more than the individual intervention. Many previous studies have suggested that HT improves mental health [4144]. Our results confirmed this effect, and show that the mental health of the participants in the GI group had significantly improved, relative to the II group, by the synergistic effect of interaction with people and plants.

The GI group showed an improved POMS vigor score in comparison with the other two groups. Additionally, the GI and II groups did not differ in the change from pre- to post-intervention. POMS is a well-established tool for assessing mood state and current emotional health. Previous studies suggest that various mood states are improved by HT $[19,45]$. In the results of the present study, the vigor score had improved, as in previous studies. Horticultural activity causes a positive change in life and mood. The results suggest that the horticultural intervention elicits positive mood changes. The GI group also showed improved salivary cortisol levels, in comparison with the other two groups, indicating that HT reduced stress. The group HT intervention reduced salivary cortisol levels more than the individual intervention. Previous studies suggest that HT reduced salivary cortisol levels, and was an effective means of stress reduction $[15,37,44]$. The group HT intervention is thought to have improved stress more than the individual intervention, as reflected in the reduction of salivary cortisol levels.

Finally, this study raises some issues for future research. This was a preliminary experiment, with a small number of participants. A possible future direction would be to conduct the study with a larger number of participants and extend those findings.

In conclusion, this study suggests that it is easier to obtain many effects of HT with a group intervention than with an individual intervention. The results of this preliminary experiment will be reexamined in a future study.

\section{Acknowledgements}

The authors would like to thank the participants and all their colleagues at the Institute of Development, Ageing and Cancer and the Tohoku University for their support. In addition, the authors thank Ms. Kana Ohkiri, Ms. Shoko Tsuzuki, Ms. Rei Takamatsu, and Ms. Mayu Fujita for assisting with psychological testing. The authors also appreciate the contribution of Mrs. Taeko Shishido, a horticultural therapist who provided advice during the preparation of the intervention program. This study was supported by a Grant-in-Aid for Young Scientists (B) (KAKENHI 24730566) from the Ministry of Education, Culture, Sports, and Science to Dr. Y.K.

\section{References}

1. Cohen S, Janicki-Deverts D, Miller GE (2007) Psychological stress and disease. JAMA 298: 1685-1687.

2. Cohen S, Herbert TB (1996) Health psychology: psychological factors and physical disease from the perspective of human psychoneuroimmunology. Annu Rev Psychol 47: 113-142.

3. Detweiler MB, Sharma T, Lane S, Kim M, Johnson BC, et al. (2010) Practitione forum: The case for using restorative natural environments in veterans' rehabilitation programs. Fed Pract. 1: 26-29.

4. Haller R, Kramer C (2006) Horticultural therapy methods: Making connections in health care, human service, and community programs. Binghamton, NY: The Haworth Press.

5. Mizuno-Matsumoto Y, Kobashi S, Hata Y, Ishikawa O, Asano F (2008). Horticultural therapy has beneficial effects on brain functions in cerebrovascular diseases. IC-MED Journal. 2: 169-182.
6. Sugihara S, Aoyama H, Sugimoto M, Takeda S, Ikeda N, et al. (2006) The psychological cognitive and immunological effects of horticultural therapy on the elderly living in a nursing home. Japanese journal of geriatric psychiatry 17: $967-975$

7. Cimprich B (1993) Development of an intervention to restore attention to cancer patients. Cancer Nurs. 12: 22-27.

8. Harzog TR, Black AM, Fountaine KA, Knott DJ (1997) Reflection and attentiona recovery as distinct benefits of restorative environments. J Environ Psychol. 17: $165-170$.

9. Porchey P (2007) Horticultural therapy: How can it make a difference in your everyday life? Proc Fla State Hort Soc. 120: 351-352

10. Ulrich RS, Parson R (1992) Influences of passive experiences with plants on individual well-being and health. The role of horticulture in human well-being and social development. Portland: Timber Press 93-105.

11. Waliczek TM, Mattson RH, Zajicek JM (1996) Benefits of community gardening to quality of life issues. J Environ Hortic 14: 204-209.

12. Rodiek S (2002) Influence of an outdoor garden on mood and stress in older persons. J Ther Hortic. 13: 13-21.

13. Langer EJ, Rodin J (1976) The effects of choice and enhanced personal responsibility for the aged: a field experiment in an institutional setting. J Pers Soc Psychol 34: 191-198.

14. Perrins-Margails N, Rugletic J, Schepis N, Stepanski H, Walsh M (2000) The immediate effects of group-based horticulture on the quality of life of persons with chronic mental illness. Occup Ther Ment Health. 16: 15-30

15. Van Den Berg AE, Custers MH (2011) Gardening promotes neuroendocrine and affective restoration from stress. J Health Psychol 16: 3-11.

16. Riordan RJ, Williams CS (1988) Gardening therapeutics for the elderly Activities, Adaptation \& Aging. 12: 103-111

17. McGinnis M (1989) Gardening as therapy for children with behavioral disorders $\mathrm{J}$ Child Adolesc Psychiatr Ment Health Nurs 2: 87-91.

18. Giglioth C, Jarrott S, Yorgason J (2004) Harvesting health: effects of three types of horticultural therapy activities for persons with dementia. Dementia 3: 161-180.

19. Wichrowski M, Whiteson J, Haas F, Mola A, Rey MJ (2005) Effects of horticultural therapy on mood and heart rate in patients participating in an inpatient cardiopulmonary rehabilitation program. J Cardiopulm Rehabil 25: 270-274.

20. Fawzy FI, Fawzy NW, Wheeler JG (1996) A post-hoc comparison of the efficiency of a psychoeducational intervention for melanoma patients delivered in group versus individual formats: An analysis of data from two studies. Psycho-Oncology 5: 81-89

21. Tasaki M, Nakane M (1997) The WHO-QOL26. Division of Mental Health and Protection of Substance Abuse, World Health Organization (in Japanese). Tokyo: Kaneko Shobou.

22. Radloff LS (1977) The CES-D scale: a self-report depression scale for research in the general population. Appl Psychol Measures 1: 385-401.

23. Shima S, Shikano T, Kitamura T, Asai M (1985) New self-rating scale for depression (in Japanese). Clin Psychiatry. 27: 717-723.

24. Fukunishi I, Wise TN, Sheridan M, Shimai S, Otake K, et al. (2001) Validity and reliability of the Japanese version of the Emotional Intelligence Scale among college students and psychiatric outpatients. Psychol Rep 89: 625-632.a

25. Fukunishi I, Wise TN, Sheridan M, Shimai S, Otake K, et al. (2001) Association of emotional intelligence with alexithymic characteristics. Psychol Rep 89: 651 658.

26. Uchiyama K, Shimai S, Utsuki N, Otake K (2001) EQS Manual. Tokyo: Jitsumukyoiku Syuppan (Practical Education Press).

27. Salovey P, Rothman AJ, Detweiler JB, Steward WT (2000) Emotional states and physical health. Am Psychol 55: 110-121.

28. Goldberg D (1972) The detection of psychiatric illness by questionnaire. London: Oxford University Press 156.

29. Nakagawa Y, Daibo I. (1966) Japanese version GHQ30 (in Japanese). Tokyo: Nihon Bunka Kagakusha. 
Citation: Kotozaki Y (2014) Comparison of the Effects of Individual and Group Horticulture Interventions. Health Care Current Reviews 2: 120. doi: $10.4172 / 2375-4273.1000120$

Page 5 of 5

30. McNair DM, Lorr M, Droppleman LF (1971) Profile of Mood States. San Diego, CA, Educational and Industrial Testing Service.

31. Yokoyama K, Araki S, Kawakami N, Tkakeshita T (1990) [Production of the Japanese edition of profile of mood states (POMS): assessment of reliability and validity]. Nihon Koshu Eisei Zasshi 37: 913-918.

32. Kotozaki Y, Kawashima R (2012) Effects of the Higashi-Nihon earthquake: posttraumatic stress, psychological changes, and cortisol levels of survivors. PLoS One 7: e34612.

33. Fukuda S, Morimoto K (2001) Lifestyle, stress and cortisol response: Review : Mental stress. Environ Health Prev Med 6: 9-14.

34. Riad-Fahmy D, Read GF, Walker RF (1983) Salivary steroid assays for assessing variation in endocrine activity. J Steroid Biochem 19: 265-272.

35. Toda M, Morimoto K, Nagasawa S, Kitamura K (2004) Effect of snack eating on sensitive salivary stress markers cortisol and chromogranin A. Environ Health Prev Med 9: 27-29.

36. O'Connor PJ, Corrigan DL (1987) Influence of short-term cycling on salivary cortisol levels. Med Sci Sports Exerc 19: 224-228.

37. Kotozaki Y (2013) The Psychological Effect of Horticultural Therapy Intervention on Earthquake-Related Stress in Women of Earthquake-Related Areas. Journal of Translational Medicine \& Epidemiology 3: 1008.
38. Barnicle T, Stoelzle Midden K (2003) The effects of a horticultural activity program on the psychological well-being of older people in a long-term care facility. Hort Technology 13: 81-85.

39. Park SH, Huh MR (2010) Effects of a horticultural program on the preschoo children's emotional intelligence and daily stress. Korean Journal of Horticultura Science \& Technology 28: 144-149.

40. Kim HY, Park YH (2010) Effect of Horticultural Therapy on the Self-Efficacy and Emotional Intelligence of Children in the Child Welfare Institutions. J Kor Soc People Plants Environ 13: 7-11.

41. Frumkin $H$ (2001) Beyond toxicity: human health and the natural environment Am J Prev Med 20: 234-240.

42. Fieldhouse $J$ (2003) The impact of an allotment group on mental health clients health, wellbeing and social networking. British Journal of Occupational Therapy 66: 286-296.

43. Page $M(2008)$ Gardening as a therapeutic intervention in mental health. Nurs Times 104: 28-30.

44. Clatworthy J, Hinds J, Camic PM (2013) Mental Health Review Journal 18 214-225.

45. Arashida E, Tsukagoshi S, Noda K, Kita T, Ohgama T, et al. (2007) Psychologica and Physiological Verification of the Therapeutic Effects of Horticultural Activity Mainly with Herbs(Human Issues \& Methodology in Horticulture). Horticultural research (Japan) 6: 491-496. 